\title{
Generalized tomographic maps
}

\author{
M. Asorey, ${ }^{1}$ P. Facchi, ${ }^{2,3}$ V.I. Man'ko, ${ }^{4}$ G. Marmo, ${ }^{5,6}$ S. Pascazio, ${ }^{7,3}$ and E.C.G. Sudarshan ${ }^{8}$ \\ ${ }^{1}$ Departamento de Física Teórica, Facultad de Ciencias, \\ Universidad de Zaragoza, 50009 Zaragoza, Spain \\ ${ }^{2}$ Dipartimento di Matematica, Università di Bari, I-70125 Bari, Italy \\ ${ }^{3}$ INFN, Sezione di Bari, I-70126 Bari, Italy \\ ${ }^{4}$ P.N. Lebedev Physical Institute, Leninskii Prospect 53, Moscow 119991, Russia \\ ${ }^{5}$ Dipartimento di Scienze Fisiche, Università di Napoli "Federico II", I-80126 Napoli, Italy \\ ${ }^{6}$ INFN, Sezione di Napoli, I-80126 Napoli, Italy \\ ${ }^{7}$ Dipartimento di Fisica, Università di Bari, I-70126 Bari, Italy \\ ${ }^{8}$ Department of Physics, University of Texas, Austin, Texas 78712, USA
}

(Dated: November 22, 2018)

\begin{abstract}
We introduce several possible generalizations of tomography for quadratic surfaces. We analyze different types of elliptic, hyperbolic and hybrid tomograms. In all cases it is possible to consistently define the inverse tomographic map. We find two different ways of introducing tomographic sections. The first method operates by deformations of the standard Radon transform. The second method proceeds by shifting a given quadric pattern. The most general tomographic transformation can be defined in terms of marginals over surfaces generated by deformations of complete families of hyperplanes or quadrics. We discuss practical and conceptual perspectives and possible applications.
\end{abstract}

PACS numbers: 03.65.Wj; 42.30.Wb; 02.30.Uu

\section{INTRODUCTION}

Most of classical applications of tomography are based on light propagation along optic rays (implicitly assumed to be straight lines). Standard Radon transform theory guarantees that a measurement of the absorption of light beams travelling in dielectric media in straight lines allows the complete reconstruction of the matter density of these media. Indeed, the original Radon transform [1] maps functions of two variables in the plane onto functions of one real variable on a line and one variable on a circle. The crucial property is that the transform is invertible and continuous [2, 3].

There exist several generalizations of the Radon transform. See, e.g., [4] and [5]. Further generalizations can be motivated by physical observations: for instance, if the function on the plane is a probability density, its Radon component is a family of probability densities of one random variable on the line, parameterized by a variable living on a circle [6]. A tomographic approach in a similar framework was applied to a free classical particle moving on a circle 7], where the phase space is a two dimensional cylinder.

In quantum mechanics the Radon transform of the Wigner function [8] was considered in the tomographic approach to the study of quantum states [9, 10] and experimentally realized with different particles and in diverse situations 11, 12, 13. . Other experiments have been proposed [14] and the whole field is in continuing evolution, also in view of its relevance in genuine quantum mechanical problems and quantum information related topics. Good reviews on recent tomographic applications can be found in Ref. 15], with emphasis on maximum likelihood estimations [16], that enable one to extract the maximum reliable information from the avail- able data.

A further development, extending the analysis to incorporate more general symplectic transforms, was presented in [17] and the mathematical mechanism at the basis of the mapping of true density states onto tomographic probabilities was elucidated in [18]. There is an interesting relation between the Radon map of Wigner functions and the formalism of star product quantization [6, 19]: symplectic tomograms are indeed the Radon components of the Wigner function and this enables one to define a procedure aimed at determining the marginal probability densities along straight lines in phase space. The knowledge of all these marginals makes possible the reconstruction of the Wigner function in the quantum case and of the probability density in the classical case.

The generalization of tomographic maps to curved surfaces opens new perspectives in the applications of tomography both to quantum and classical systems. Some attempts to study marginals along curves other than straight lines were introduced in Ref. [20]. Very recently, optical "accelerating" Airy beams were observed [21]: these beams could be used to perform a tomographic map over parabolas in phase space. A generalization of tomography to this kind of applications requires a generalization of the Radon transform.

The aim of this article is to study generalizations of the Radon transform to multidimensional phase spaces and to marginals along curves or surfaces. Most of the generalizations of the Radon transform proceed by considering geodesic submanifolds of a given Riemannian manifold. We develop here a different approach, that can be applied to the Radon components of the probability densities of classical particles in phase space, and construct the corresponding tomographic maps.

This article is organized as follows. In Sec. II we re- 


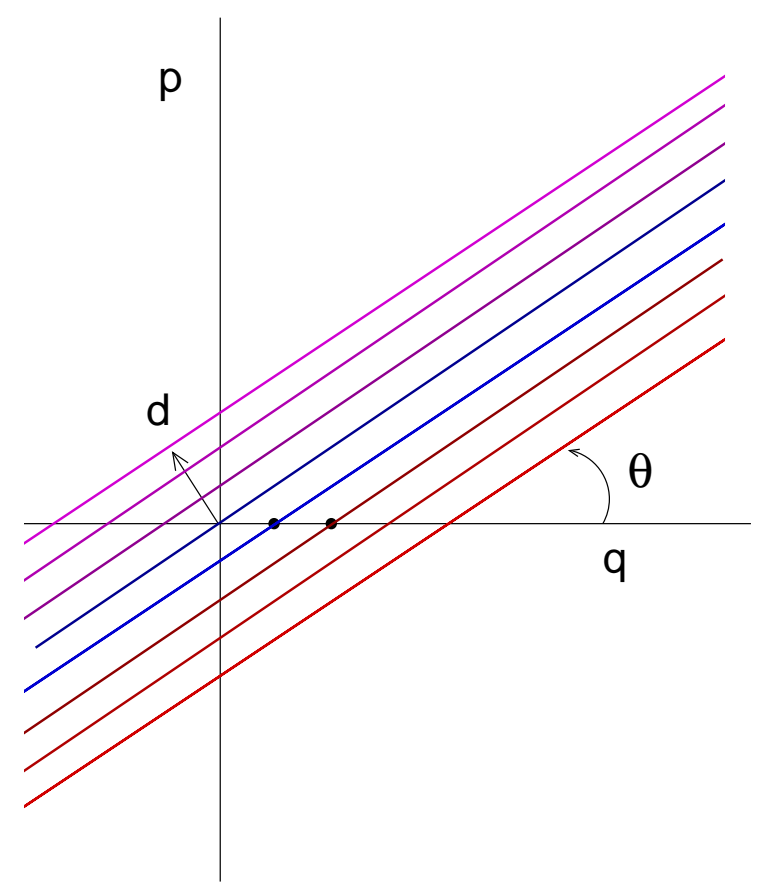

FIG. 1: Tomography on the plane; $(q, p) \in \mathbb{R}^{2}, d \in \mathbb{R}, \theta \in \mathbb{S}$ (unit circle).

view the standard tomographic application of the Radon transform on the plane. In Sec. III] we consider the generalization to arbitrary dimensions. A deformation of the Radon transform with applications to elliptic and hyperbolic problems is presented in Sec. IV In Sec. V we introduce a new type of transform involving hyperbolic, elliptic and parabolic quadrics. The transform is defined by translations of a basic pattern. Finally in Sec. VI we discuss the relevance of our results for future applications.

\section{TOMOGRAPHY ON THE PLANE}

Let us consider a function $f(q, p)$ on the phase space $(q, p) \in \mathbb{R}^{2}$ of a particle moving on the line $q \in \mathbb{R}$. The Radon transform, in its original formulation, solves the following problem: reconstruct a function of two variables, say $f(p, q)$, from its integrals over arbitrary lines.

In the $(q, p)$ plane, a line is given by the equation

$$
X-\mu q-\nu p=0,
$$

with $(\mu, \nu) \neq(0,0)$. Thus, the family of lines has the manifold structure $\mathbb{R} \times \mathbb{S}$, with $\mathbb{S}$ the unit circle, $d=$ $X / \sqrt{\mu^{2}+\nu^{2}} \in \mathbb{R}$ and $\mu / \nu=\tan \theta, \theta \in \mathbb{S}$ (see Fig. 10).

It is possible to write the Radon transform in affine language (tomographic map) [1, [5]

$$
\begin{aligned}
\omega_{f}(X, \mu, \nu) & =\langle\delta(X-\mu q-\nu p)\rangle \\
& =\int_{\mathbb{R}^{2}} f(q, p) \delta(X-\mu q-\nu p) d q d p,
\end{aligned}
$$

where $\delta$ is the Dirac function and the parameters $X, \mu, \nu \in \mathbb{R}$.

The inverse transform of (2) reads

$$
f(q, p)=\int_{\mathbb{R}^{3}} \omega_{f}(X, \mu, \nu) e^{i(X-\mu q-\nu p)} \frac{d X d \mu d \nu}{(2 \pi)^{2}} .
$$

The homogeneity of $\omega_{f}(X, \mu, \nu)$

$$
\omega_{f}(\lambda X, \lambda \mu, \lambda \nu)=\frac{1}{|\lambda|} \omega_{f}(X, \mu, \nu),
$$

$\forall \lambda \in \mathbb{R}, \lambda \neq 0$, is a direct consequence of (2). If the function $f(q, p)$ is a probability density function on the phase space of a classical particle, i.e.

$$
f(q, p) \geq 0, \quad \int_{\mathbb{R}^{2}} f(q, p) d q d p=1,
$$

the function $\omega_{f}(X, \mu, \nu)$ is also nonnegative and is called symplectic tomogram or Radon transform of the distribution function $f(q, p)$ (in analogy to the Fourier transform of a function). The Radon transform contains the same information on the state of the particle evolving on the phase space as the initial distribution function. In summary, the tomograms

$$
\omega_{f}(X, \mu, \nu) \geq 0, \quad \int_{\mathbb{R}} \omega_{f}(X, \mu, \nu) d X=1, \quad \forall \mu, \nu,
$$

form a family of density functions that depends on the two real parameters $\mu$ and $\nu$.

\section{TOMOGRAMS ON HYPERPLANES}

The above construction can be generalized to higher dimensional spaces in a straightforward way. Let us consider a function $f(q)$ on the $n$-dimensional space $q \in \mathbb{R}^{n}$. Is it possible to reconstruct the function $f$ from its integrals over arbitrary $(n-1)$-dimensional linear submanifolds? The answer to this question is positive and provides a generalization of the original Radon transform.

A generic hyperplane is given by the equation

$$
X-\mu \cdot q=0,
$$

with $X \in \mathbb{R}$ and $\mu \in \mathbb{R}^{n}$. Due to homogeneity, this family of hyperplanes is an $n$-dimensional manifold diffeomorphic to $\mathbb{R} \times \mathbb{S}^{n-1}$, because any hyperplane can be characterized by its unit normal vector $\mu /|\mu|$ and its distance to the origin $|X| /|\mu|$. Note that this manifold is not diffeomorphic to $\mathbb{R}^{n}$ because the sphere $\mathbb{S}^{n-1}$ is compact.

The Radon transform is given by

$$
\begin{aligned}
\omega_{f}(X, \mu) & =\langle\delta(X-\mu \cdot q)\rangle \\
& =\int_{\mathbb{R}^{n}} f(q) \delta(X-\mu \cdot q) d^{n} q .
\end{aligned}
$$

When $n=2$ Eq. (2) is recovered. 


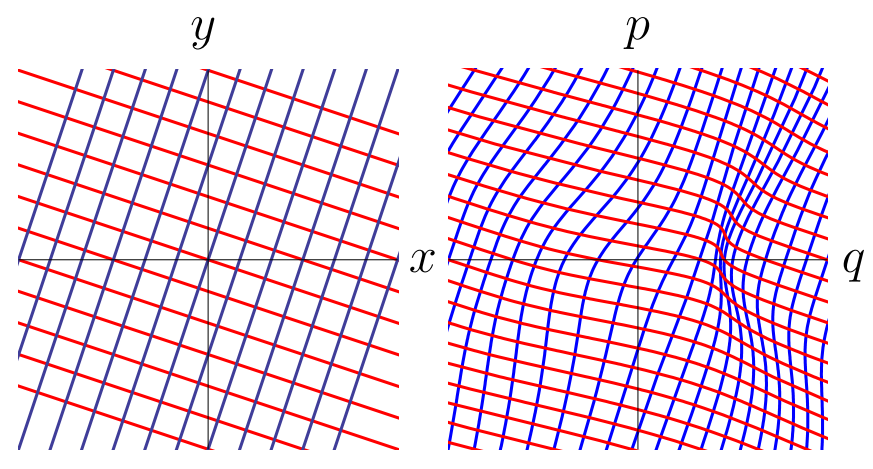

FIG. 2: Diffeomorphism of the plane: $(q, p) \in \mathbb{R}^{2} \rightarrow(x, y)=$ $\varphi(q, p) \in \mathbb{R}^{2}$.

The inverse transform of (8) reads

$$
f(q)=\int_{\mathbb{R}^{n+1}} \omega_{f}(X, \mu) e^{i(X-\mu \cdot q)} \frac{d X d^{n} \mu}{(2 \pi)^{n}} .
$$

The homogeneity of $\omega_{f}(X, \mu)$

$$
\omega_{f}(\lambda X, \lambda \mu)=\frac{1}{|\lambda|} \omega_{f}(X, \mu)
$$

$\forall \lambda \in \mathbb{R}, \lambda \neq 0$, is a direct consequence of (8). If the function $f(q)$ is a probability density function on $\mathbb{R}^{n}$

$$
f(q) \geq 0, \quad \int_{\mathbb{R}^{n}} f(q) d^{n} q=1,
$$

also the tomograms $\omega_{f}(X, \mu)$ are probability densities

$$
\omega_{f}(X, \mu) \geq 0, \quad \int_{\mathbb{R}} \omega_{f}(X, \mu) d X=1, \quad \forall \mu \in \mathbb{R}^{n}
$$

and the family of tomograms depends on the $n$ real parameters $\mu$. In quantum mechanics such construction was applied to Wigner functions providing a center of mass tomography [22].

\section{TOMOGRAMS ON HYPERSURFACES}

A simple mechanism that allows nonlinear generalizations of the Radon transform is the combination of the standard transform with a diffeomorphism of the underlying $\mathbb{R}^{n}$ space. Let us consider a function $f(q)$ on the $n$-dimensional space $q \in \mathbb{R}^{n}$. The problem is to reconstruct $f$ from its integrals over an $n$-parameter family of submanifolds of codimension one.

We can construct such a family by diffeomorphic deformations of the hyperplanes (in the $x \in \mathbb{R}^{n}$ space)

$$
X-\mu \cdot x=0,
$$

with $X \in \mathbb{R}$ and $\mu \in \mathbb{R}^{n}$. Let us consider a diffeomorphism of $\mathbb{R}^{n}$

$$
q \in \mathbb{R}^{n} \mapsto x=\varphi(q) \in \mathbb{R}^{n}
$$

The hyperplanes (13) are deformed by $\varphi$ into a family of submanifolds (in the $q$ space)

$$
X-\mu \cdot \varphi(q)=0 \text {. }
$$

The case $n=2$ is displayed in Fig. 2. $(q, p) \in \mathbb{R}^{2} \rightarrow$ $(x, y)=\varphi(q, p) \in \mathbb{R}^{2}$.

Given a probability density $\tilde{f}(x)$ on the $x$ space, the Radon transform can be rewritten as

$$
\begin{aligned}
\omega_{f}(X, \mu) & =\langle\delta(X-\mu \cdot x)\rangle \\
& =\int_{\mathbb{R}^{n}} \tilde{f}(x) \delta(X-\mu \cdot x) d^{n} x \\
& =\int_{\mathbb{R}^{n}} \tilde{f}(\varphi(q)) \delta(X-\mu \cdot \varphi(q)) J(q) d^{n} q,
\end{aligned}
$$

where

$$
J(q)=\left|\frac{\partial x_{i}}{\partial q_{j}}\right|=\left|\frac{\partial \varphi_{i}(q)}{\partial q_{j}}\right|
$$

is the Jacobian of the transformation.

Observe now that

$$
\tilde{f}(x) d^{n} x=\tilde{f}(\varphi(q)) J(q) d^{n} q,
$$

whence $f(q)=\tilde{f}(\varphi(q)) J(q)$ is a probability density. Therefore the tomograms are given by

$$
\begin{aligned}
\omega_{f}(X, \mu) & =\langle\delta(X-\mu \cdot \varphi(q))\rangle \\
& =\int_{\mathbb{R}^{n}} f(q) \delta(X-\mu \cdot \varphi(q)) d^{n} q,
\end{aligned}
$$

with $X \in \mathbb{R}$ and $\mu \in \mathbb{R}^{n}$.

The inverse transform follows by (9):

$$
\begin{aligned}
f(q) & =\tilde{f}(\varphi(q)) J(q) \\
& =\int_{\mathbb{R}^{n+1}} \omega_{f}(X, \mu) J(q) e^{i(X-\mu \cdot \varphi(q))} \frac{d X d^{n} \mu}{(2 \pi)^{n}},
\end{aligned}
$$

with a modified kernel

$$
K(q ; X, \mu)=J(q) e^{i(X-\mu \cdot \varphi(q))}=\left|\frac{\partial \varphi_{i}(q)}{\partial q_{j}}\right| e^{i(X-\mu \cdot \varphi(q))} .
$$

Therefore, a probability density distribution on $\mathbb{R}^{n}$

$$
f(q) \geq 0, \quad \int_{\mathbb{R}^{n}} f(q) d^{n} q=1
$$

produces tomograms $\omega_{f}(X, \mu)$ that are probability densities

$$
\omega_{f}(X, \mu) \geq 0, \quad \int_{\mathbb{R}} \omega_{f}(X, \mu) d X=1, \quad \forall \mu \in \mathbb{R}^{n} .
$$

The family of tomograms depends on the $n$ real parameters $\mu$. We can now consider different applications of these deformed generalizations of the Radon transform. 


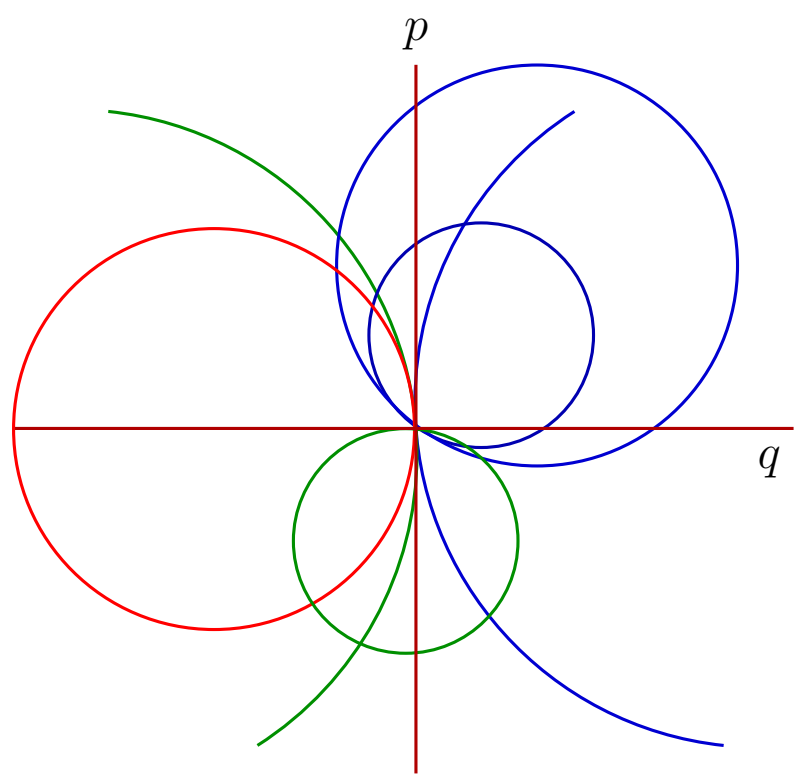

FIG. 3: Deformed circular tomography. All circles pass through the origin.

\section{A. Circles in the plane}

In the punctured $(x, y)$ plane without the origin $(0,0)$, the conformal inversion

$$
(x, y)=\varphi(q, p)=\left(\frac{q}{q^{2}+p^{2}}, \frac{p}{q^{2}+p^{2}}\right),
$$

maps the family of lines

$$
X-\mu x-\nu y=0
$$

into a family of circles

$$
X\left(q^{2}+p^{2}\right)-\mu q-\nu p=0,
$$

centered at

$$
C=\left(\frac{\mu}{2 X}, \frac{\nu}{2 X}\right)
$$

and passing through the origin (see Fig. 33). When $X=0$ they degenerate into lines through the origin.

The Jacobian reads

$$
J(q, p)=\left|\frac{\partial(x, y)}{\partial(q, p)}\right|=\frac{1}{\left(q^{2}+p^{2}\right)^{2}},
$$

whence the transformation is a diffeomorphism of the punctured plane of functions $f \in L^{1}\left(\mathbb{R}^{2}\right)$. The singularity of the transformation at the origin $(0,0)$ is irrelevant for tomographic integral transformations because it only affects a zero measure set.

Equations (19)-201) become

$$
\begin{aligned}
& \omega_{f}(X, \mu, \nu)=\left\langle\delta\left(X-\frac{\mu q}{q^{2}+p^{2}}-\frac{\nu p}{q^{2}+p^{2}}\right)\right\rangle \\
& =\int_{\mathbb{R}^{2}} f(q, p) \delta\left(X-\frac{\mu q}{q^{2}+p^{2}}-\frac{\nu p}{q^{2}+p^{2}}\right) d q d p
\end{aligned}
$$

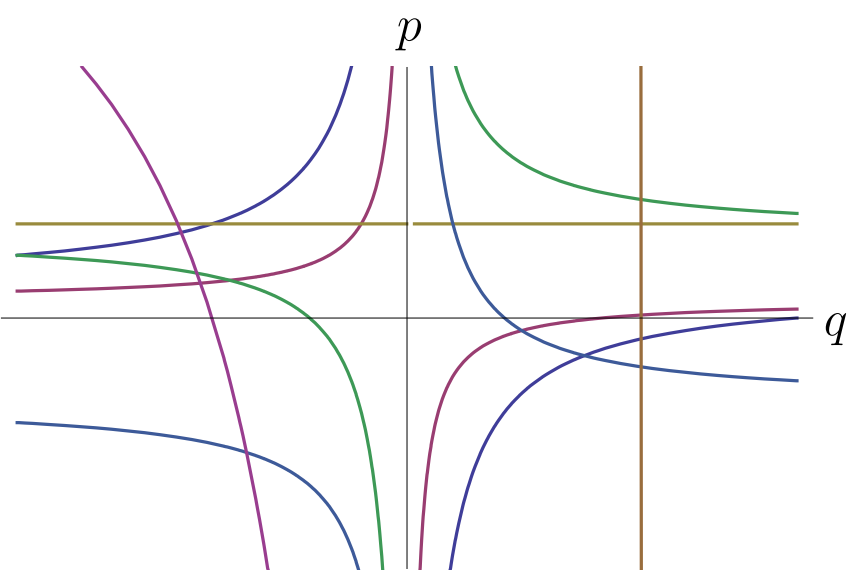

FIG. 4: Hyperbolic tomography.

and

$f(q, p)=\int_{\mathbb{R}^{3}} \omega_{f}(X, \mu) \frac{e^{i\left(X-\frac{\mu q}{q^{2}+p^{2}}-\frac{\nu p}{q^{2}+p^{2}}\right)}}{(2 \pi)^{2}\left(q^{2}+p^{2}\right)^{2}} d X d \mu d \nu$.

\section{B. Hyperbolas in the plane}

In the $(x, y)$ plane the family of lines

$$
X-\mu x-\nu y=0
$$

is mapped into a family of hyperbolas

$$
X-\frac{\mu}{q}-\nu p=0,
$$

with asymptotes

$$
q=0, \quad p=\frac{X}{\nu},
$$

by the transformation

$$
(x, y)=\varphi(q, p)=\left(\frac{1}{q}, p\right) .
$$

For $\mu>0$ the hyperbolas are in the second and fourth quadrants, while for $\mu<0$ they are in the first and third quadrants (see Fig. (4). When $\mu=0$ or $\nu=0$ they degenerate into horizontal or vertical lines, respectively.

The Jacobian reads

$$
J(q, p)=\left|\frac{\partial(x, y)}{\partial(q, p)}\right|=\frac{1}{q^{2}},
$$

whence the transformation is a diffeomorphism in the cut plane without the axis $(0, y)$.

Equations (19)- (20) become

$$
\begin{aligned}
\omega_{f}(X, \mu, \nu) & =\left\langle\delta\left(X-\frac{\mu}{q}-\nu p\right)\right\rangle \\
& =\int_{\mathbb{R}^{2}} f(q, p) \delta\left(X-\frac{\mu}{q}-\nu p\right) d q d p
\end{aligned}
$$


and

$$
f(q, p)=\int_{\mathbb{R}^{3}} \omega_{f}(X, \mu) \frac{1}{q^{2}} e^{i\left(X-\frac{\mu}{q}-\nu p\right)} \frac{d X d \mu d \nu}{(2 \pi)^{2}} .
$$

The tomograms (19), (29) and (36) have the homogeneity property (10).

\section{Hyperboloids in $\mathbb{R}^{n}$}

The generalization to higher dimensions of tomographic maps that can be given in terms of quadratic expressions is straightforward. Let us consider for example the following tomographic map

$\omega_{f}(X, \mu, \nu)=\int_{\mathbb{R}^{2 n}} \delta(X-\mu \cdot q-\nu(q, p)) f(q, p) d^{n} q d^{n} p$,

where $p$ and $q$ are vectors in $\mathbb{R}^{n}$ and

$$
\nu(q, p)=\sum_{j=1}^{n} \nu_{j} q_{j} p_{j}
$$

This map corresponds to a deformation of the standard multidimensional Radon transform by means of the following diffeomorphism of $\mathbb{R}^{2 n} \backslash \bigcup_{j}\left\{(q, p): q_{j}=0\right\}$

$$
\left(q_{i}, p_{j}\right) \mapsto\left(x_{i}, y_{j}\right)=\left(q_{i}, q_{j} p_{j}\right),
$$

whose Jacobian is

$$
J(q, p)=\left|\frac{\partial(x, y)}{\partial(q, p)}\right|=\prod_{j=1}^{n}\left|q_{j}\right| .
$$

The inverse map is given by

$$
\begin{array}{r}
f(q, p)=\int_{\mathbb{R}^{2 n+1}} \frac{d X d^{n} \mu d^{n} \nu}{(2 \pi)^{2 n}} \omega_{f}(X, \mu, \nu) \\
\times \prod_{j=1}^{n}\left|q_{j}\right| \mathrm{e}^{i(X-\mu \cdot q-\nu(q, p))} .
\end{array}
$$

This corresponds to the higher dimensional generalization of the Bertrand-Bertrand tomography [9].

Note that when $n=2$, by interchanging the role of $X$ and $-\mu$, one recovers the same distribution of hyperbolas in the plane analyzed in the previous subsection IVB

Although the above generalizations might be very useful for light rays tomograms, all of them involve integration over unbounded submanifolds. One would like to generalize the Radon transform to marginals defined over compact submanifolds, that are bounded on a compact domain around $(p, q)$. This case will be investigated in the following section.

\section{TOMOGRAMS ON QUADRICS}

Let us now look for a different generalization of tomograms. We shall consider marginals along compact quadrics. This can be achieved by shifting and scaling a given quadric pattern

$$
X=(q-\mu, B(q-\mu)),
$$

where $B$ is a non-degenerate symmetric operator with respect to the scalar product $(x, y)=x \cdot y$. A new generalization of the tomographic map can be defined by

$\omega_{f}(X, \mu ; B)=\int_{\mathbb{R}^{n}} f(q) \delta(X-(q-\mu, B(q-\mu))) d^{n} q$.

Eq. (44) defines a completely different type of transform, supported on the quadrics defined by equation (43). It is easy to show that the inverse map is defined by

$$
\begin{gathered}
f(q)=\frac{|\operatorname{det} B|}{\pi^{n}} \int_{\mathbb{R}^{n+1}} d X d^{n} \mu \omega_{f}(X, \mu ; B) \\
\times \mathrm{e}^{i(X-(q-\mu, B(q-\mu)))} .
\end{gathered}
$$

Indeed, by applying the definition of tomographic map (45)

$$
\begin{aligned}
& \frac{|\operatorname{det} B|}{\pi^{n}} \int d X d^{n} \mu \mathrm{e}^{i(X-(q-\mu, B(q-\mu)))} \omega_{f}(X, \mu ; B) \\
& =\frac{|\operatorname{det} B|}{\pi^{n}} \int d X d^{n} \mu \mathrm{e}^{i(X-(q-\mu, B(q-\mu)))} \\
& \quad \times \int d^{n} \xi \delta(X-(\xi-\mu, B(\xi-\mu))) f(\xi),
\end{aligned}
$$

which after integration over $X$ yields

$$
\begin{aligned}
& \frac{|\operatorname{det} B|}{\pi^{n}} \int d^{n} \xi f(\xi) \\
& \times \int d^{n} \mu \mathrm{e}^{i((\xi-\mu, B(\xi-\mu))-(q-\mu, B(q-\mu)))} \\
= & \frac{|\operatorname{det} B|}{\pi^{n}} \int d^{n} \xi d^{n} \mu f(\xi) \mathrm{e}^{i[(\xi, B \xi)-(q, B q)+2(q-\xi, B \mu)]} \\
= & \int d^{n} \xi f(\xi) \mathrm{e}^{i[(\xi, B \xi)-(q, B q)]} \delta^{n}(q-\xi)=f(q) .
\end{aligned}
$$

The meaning of the above tomographic map depends on the features of $B$. If we assume that $B$ is strictly positive (elliptic case), this map corresponds to averages of $f$ along the ellipsoids defined by Eq. (43). In particular if all the eigenvalues of $B$ are equal to $b^{2}$ it corresponds to integration over spheres centered at $\mu$ of (squared) radius $X / b^{2}$, namely,

$$
b^{2}(q-\mu)^{2}=X . \quad(X>0)
$$

Note that, in the two dimensional case, the distribution of circles is different from that obtained by the transform defined by diffeomorphisms in Sec. IV] There, the 


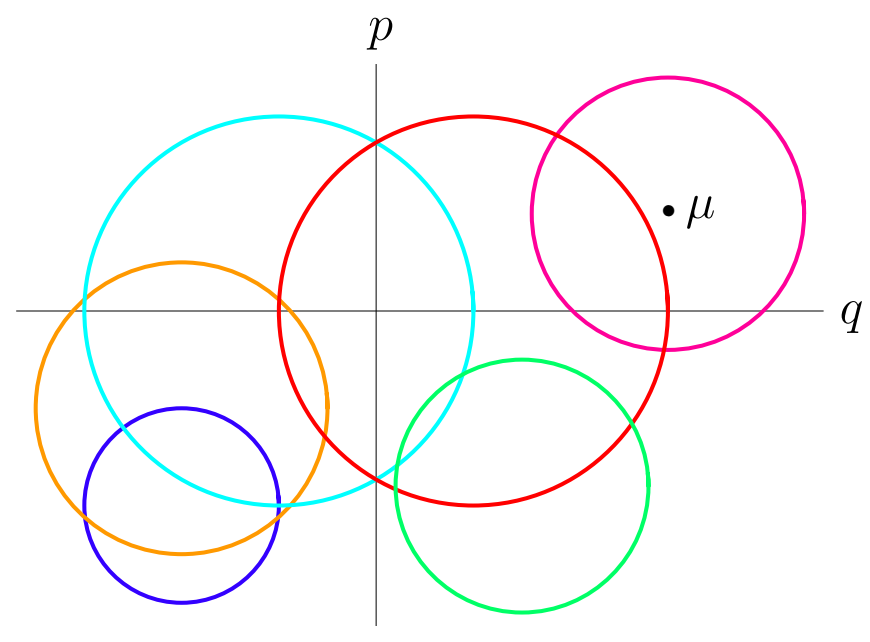

FIG. 5: Tomography on circles of center $\mu$ and (squared) radius $X / b^{2}$ on the plane.

family of tomograms were defined only on circles passing through the origin, including straight lines (circles of infinite radius). Here, we are taking into account all possible circles of finite radius in the plane (see Fig. 5). This corresponds to trajectories of particles moving in a plane under the action of a constant magnetic field. From a practical perspective, this new tomographic map would make possible a different practical implementation of tomography.

When $B$ has both positive and negative eigenvalues this correspond to hyperbolic tomography with averages of $f$ along the hyperboloids defined by Eq. (43), e.g.,

$$
b^{2}\left(q_{1}-\mu_{1}\right)^{2}-c^{2}\left(q_{2}-\mu_{2}\right)^{2}=X .
$$

In the case of degenerated $B$ forms we have to consider a hybrid transform. $B$ can then be decomposed into a non-degenerated bilinear form and a linear form. In this case the tomography of the linear components should be treated as the standard Radon transform, whereas the non-degenerate variables should transform as above. Let us consider for example a simple three-dimensional case with

$$
\bar{B}=\left(\begin{array}{lll}
1 & 0 & 0 \\
0 & 1 & 0 \\
0 & 0 & 0
\end{array}\right)
$$

In this case we can define the following tomographic map

$$
\begin{aligned}
& \omega_{f}(X, \mu ; \bar{B})=\int_{\mathbb{R}^{3}} d^{3} q f(q) \\
& \quad \times \delta\left(X-\left(q_{1}-\mu_{1}\right)^{2}-\left(q_{2}-\mu_{2}\right)^{2}-\mu_{3} q_{3}\right),
\end{aligned}
$$

with inverse transform

$$
\begin{aligned}
f(q)= & \int_{\mathbb{R}^{4}} \frac{d X d^{3} \mu}{2 \pi^{3}} \omega_{f}(X, \mu ; \bar{B}) \\
& \times \mathrm{e}^{i\left(X-\left(q_{1}-\mu_{1}\right)^{2}-\left(q_{2}-\mu_{2}\right)^{2}-\mu_{3} q_{3}\right)} .
\end{aligned}
$$

\section{CONCLUSIONS AND PERSPECTIVES}

Let us discuss the main findings of this article, both from mathematical and physical perspectives. From a mathematical viewpoint, the generalizations of the Radon transform introduced here enable one to consider marginals defined over submanifolds that are not necessarily geodesic submanifolds in Riemannian spaces or Lagrangian submanifolds of symplectic manifolds. These transforms define tomograms over compact submanifolds and can be more suitable for physical applications, because the practical implementation of a tomogram can only achieved in local terms. In this new framework the recovery of a local value (of a probability distribution on phase space in the classical theory, or of a Wigner distribution in the quantum case) only involves integration over manifolds that do not reach infinity. In a previous article [7], we considered the tomography of a classical particle moving on a circle, which required the definition of marginals over the helices on a cylinder. Now, in the light of the new transforms just introduced, we have the possibility of performing tomography over compact submanifolds even for classical systems that evolve in unbounded domains. This is a significant conceptual step forward.

Physically, the new reconstruction formulas enable one to generalize the measurement procedures of the matter density of an object. In a material medium with a strongly inhomogeneous refractive index, the radiation beams (light beams, sonic beams or matter waves) would propagate along curved lines and yet yield complete information on the matter distribution by means of generalized Radon transforms. For illustrative purposes our examples focus on two-dimensional situations [see e.g. Eqs. (24), (34) and (49)] but the approach we propose is more general and valid in $\mathbb{R}^{n}$.

In quantum optics the new "non-linear" Radon transforms can be easily extended to the quantum domain by using the Weyl-Wigner map. This will be discussed in a future publication. The results of this article show that the reconstruction of the Wigner function using optical or symplectic tomography based on straight-line Radon transform can be extended to situations in which the marginals in phase space are measured for curved hyperbolas or ellipses. In particular, parabolic tomography could be implemented with the recently observed accelerated Airy beams [21].

Novel physical applications of tomography have attracted increasing attention during the last few years. Recent applications involving neutrinos, e.g. to get a mapping of the Earth inner density [23], do not require new concepts of tomographic maps. However, neutrino tomography of gamma ray bursts and massive stellar collapses [24] might require generalized tomography. In particular, gamma rays tomography that made possible the discovery of asphericity in supernovae explosions [25] or imaging of astrophysical sources [26] can involve nonlinear trajectories of gamma rays due to strong gravi- 
tational lensing effects. In those cases, generalizations of tomographic maps like the ones considered in this paper are necessary.

\section{Acknowledgments}

We thank J.F. Cariñena and F. Ventriglia for helpful discussions. V.I.M. was partially supported by Italian INFN and thanks the Physics Department of the Uni- versity of Naples for the kind hospitality. P.F. and S.P. acknowledge the financial support of the European Union through the Integrated Project EuroSQIP. The work of M.A. and G.M. was partially supported by a cooperation grant INFN-CICYT. M.A. was also partially supported by the Spanish CICYT grant FPA2006-2315 and DGIIDDGA (grant2006-E24/2). The work of V.I.M. was partially supported by the Russian Foundation for Basic Research under Project No. 07-02-00598.
[1] J. Radon, Über die bestimmung von funktionen durch ihre integralwerte längs dewisse mannigfaltigkeiten, Breichte Sachsische Akademie der Wissenschaften, Leipzig, Mathematische-Physikalische Klasse, 69 S. 262 (1917).

[2] F. John, Plane waves and spherical means: Applied to Partial Differential Equations (Wiley Interscience, New York, 1955).

[3] R. S. Strichartz, American Mathematical Monthly 89, 377 (1982).

[4] S. Helgason, Ann. of Math. 98, 451 (1973); Groups and Geometric Analysis (Academic Press, Orlando, 1984); The Radon Transform (Birkhauser, Boston, 1980).

[5] I. M. Gel'fand and G. E. Shilov, Generalized Functions: Properties and Operations, Vol. 5 (Academic Press, 1966).

[6] O. V. Man'ko and V. I. Man'ko, J. Russ. Laser Res. 18, 407 (1997).

[7] M. Asorey, P. Facchi, V. I. Man'ko, G. Marmo, S. Pascazio and E. C. G. Sudarshan, Phys. Rev. A 76, 012117 (2007).

[8] E. P. Wigner, Phys. Rev. 40, 749 (1932); J Moyal, Proc. Camb. Phil. Soc. 45, 99 (1949); M. Hillary, R. F. O'Connell, M. O. Scully, and E. Wigner, Phys. Rep. 106, 121 (1984).

[9] J. Bertrand and P. Bertrand, Found. Phys. 17, 397 (1987).

[10] K. Vogel and H. Risken, Phys. Rev. A 40, 2847 (1989).

[11] D. T. Smithey, M. Beck, M. G. Raymer, and A. Faridani, Phys. Rev. Lett. 70, 1244 (1993).

[12] G. Zambra, A. Andreoni, M. Bondani, M. Gramegna, M. Genovese, G. Brida, A. Rossi, and M. G. A. Paris, Phys. Rev. Lett. 95, 063602 (2005); M. Genovese, G. Brida, M. Gramegna, M. Bondani, G. Zambra, A. Andreoni, A.R. Rossi, M.G.A. Paris, Laser Physics 16, 385 (2006); G.
Brida, M. Genovese, F. Piacentini, Matteo G. A. Paris, Optics Letters, 31, 3508 (2006).

[13] C. Kurtsiefer, T. Pfau, and J. Mlynek, Nature 386, 150 (1997).

[14] G. Badurek, et al, Phys. Rev. A 73, 032110 (2006).

[15] Quantum State Estimation, Lecture Notes in Physics Vol. 649, edited by M. G. A. Paris and J. Řeháček (Springer, Berlin, 2004).

[16] P. Banáš, J. Řeháček, and Z. Hradil, Phys. Rev. A 74, 014101 (2006); Z. Hradil, D. Mogilevtsev, and J. Řeháček, Phys. Rev. Lett. 96, 230401 (2006).

[17] S. Mancini, V. I. Man'ko and P. Tombesi, Quantum Semiclass. Opt. 7, 615 ( 1995).

[18] V. I. Man'ko, G. Marmo, A. Simoni and F. Ventriglia, Open Sys. \& Information Dyn. 13, 239 (2006).

[19] O. V. Man'ko, V. I. Man'ko and G. Marmo, Phys. Scr. 62, 446 (2000).

[20] M. A. Man'ko, V. I. Man'ko and R. V. Mendes, J. Phys. A 34, 8321 (2001).

[21] G. A. Siviloglou, J. Broky, A. Dogariu and D. N. Christodoulides, Phys. Rev. Lett. 99, 213901 (2007).

[22] A. S. Arkhipov and V. I. Man'ko, Phys. Rev. A 71, 012101 (2005).

[23] C. Kuo, H.J. Crawford, R. Jeanloz, B. Romanowicz, G. Shapiro and M. L. Stevenson, Earth and Planetary Science Letters 133 (1995) 95.

[24] S. Razzaque, P. Meszaros and E. Waxman, Phys. Rev. D 68, 3001 (2003).

[25] K. Maeda et al., Science Express (online edition of Science), 31 January 2008.

[26] S. N. Zhang, G. J. Fishman, B. A. Harmon and W. S. Paciesas, Nature 366245 (1993). 